Théologiques

Théologiques

\title{
Dualisme et construction de l'identité sectaire à Qumrân
}

\section{Jean Duhaime}

Volume 13, numéro 1, printemps 2005

Croire et croyances

URI : https://id.erudit.org/iderudit/012524ar

DOI : https://doi.org/10.7202/012524ar

Aller au sommaire du numéro

\section{Éditeur(s)}

Faculté de théologie et de sciences des religions, Université de Montréal

\section{ISSN}

1188-7109 (imprimé)

1492-1413 (numérique)

Découvrir la revue

\section{Citer cet article}

Duhaime, J. (2005). Dualisme et construction de l'identité sectaire à Qumrân. Théologiques, 13(1), 43-57. https://doi.org/10.7202/012524ar

\section{Résumé de l'article}

Plusieurs textes de Qumrân sont fortement imprégnés d'une idéologie dualiste. Empruntant aux théories et modèles des sciences sociales, cette étude examine la contribution d'une telle idéologie à la structuration de l'identité sectaire dans le ou les groupes dont ils sont le reflet. Après des précisions sur le concept d'univers symbolique et la place du dualisme qumranien dans ce cadre général, l'auteur analyse sommairement les règles d'admission, l'opposition entre le pur et l'impur et l'affrontement des deux esprits selon la Règle de la communauté , les récits identitaires des Hymnes et la représentation du combat ultime de la lumière contre les ténèbres dans la Règle de la Guerre .
Tous droits réservés @ Faculté de théologie et de sciences des religions, Université de Montréal, 2006
Ce document est protégé par la loi sur le droit d'auteur. L’utilisation des services d'Érudit (y compris la reproduction) est assujettie à sa politique d'utilisation que vous pouvez consulter en ligne.

https://apropos.erudit.org/fr/usagers/politique-dutilisation/ 


\title{
Dualisme et construction de l'identité sectaire à Qumrân
}

\author{
Jean Duhaime \\ Faculté de théologie et de sciences des religions \\ Université de Montréal
}

\section{Introduction}

Quelques-uns des textes les plus connus de la bibliothèque de Qumrân (la Règle de la communauté, les Hymnes, la Règle de la guerre ${ }^{1}$, etc.) sont fortement imprégnés d'une idéologie sectaire dualiste. Le monde et l'être humain y apparaissent comme le théâtre d'un combat à finir entre la lumière et les ténèbres, ou entre la vérité et la perversion. Comment et pourquoi un groupe religieux élabore-t-il de telles croyances? À quoi lui servent-elles ? Y a-t-il un lien entre ces innovations ou recompositions religieuses de type idéologique et la situation historique, sociale ou politique de ceux qui les produisent ? L'historien des religions et le théologien s'intéresseront surtout à saisir l'origine et le contenu de ces croyances pour en dégager la genèse, la spécificité et l'influence; le sociologue ou l'anthropologue verra plutôt leur élaboration et leur intégration dans la vie d'une communauté comme des moyens qu'un ou plusieurs groupes juifs anciens ont mis en œuvre pour se doter d'une identité particulière et pour la consolider grâce à un ensemble de stratégies complémentaires.

En adoptant cette dernière perspective, je me propose d'explorer ici quelques facettes du dualisme qumranien tel qu'il se donne à voir dans des textes et thèmes particulièrement représentatifs. Après avoir précisé la notion d'univers symbolique et situé le dualisme qumranien dans ce cadre général, j'analyse sommairement quelques descriptions des règles d'admission (le

1. Ces textes sont disponibles en français dans les traductions de Carmignac et al. (1961-1963), de Dupont-Sommer et Philonenko (1987) et de Wise, Abegg et Cook (2001). Pour la Règle de la communauté, sauf indication contraire, j'utilise la traduction que j'ai publiée dans Charlesworth 1996, 91-103. 
«passage » dans l'alliance), des enseignements portant sur le pur et l'impur, puis sur l'affrontement des deux esprits, avant de me tourner vers le genre de récit identitaire proposé par les Hymnes et vers la représentation du combat ultime entre les forces du bien et celles du mal (la guerre eschatologique). À chaque fois, je m'appuie sur des théories et modèles empruntés aux sciences sociales pour examiner la contribution des croyances exprimées dans ces textes à la structuration de l'identité sectaire du ou des groupes dont elles sont le reflet ${ }^{2}$.

\section{Un univers symbolique sectaire}

Pour Peter L. Berger et Thomas Luckmann (1996), ce que les membres d'une même société conçoivent comme la réalité quotidienne la plus banale est, en fait, le résultat d'un ensemble d'interprétations subjectives dont ils sont les auteurs et qu'ils se transmettent et intériorisent à travers des processus de socialisation primaire ou secondaire. Cette réalité est constamment justifiée et légitimée par son objectivation dans le langage, sa formulation en maximes ou proverbes, la constitution de corpus de connaissances propres à divers secteurs d'activités et, ultimement, par l'élaboration d' "univers symboliques » où l'ensemble des réalités et des expériences humaines sont intégrées en un tout cohérent.

Les univers symboliques sont à leur tour maintenus par le recours à des «machineries conceptuelles » telles que la mythologie, la théologie, la philosophie ou la science. Ils ne sont cependant jamais définitifs et peuvent être constamment remis en cause, particulièrement au moment de crises ou de transformations culturelles et sociales. De nouvelles interprétations de la réalité peuvent alors se développer, entrer en concurrence et conduire à la formation de groupes marginaux, s'isolant dans leur vérité.

Du point de vue sociologique, la «secte » apparaît comme la résultante d'une telle dynamique. Comme le souligne Jutta M. Jokiranta (2001), une secte peut être caractérisée par la tension qu'elle entretient avec son environnement socioculturel, sa prétention exclusive à la légitimité et sa tendance à délimiter nettement ses frontières. Au plan du discours, cela se traduit par une rhétorique polémique qui incite à se couper du monde extérieur.

2. Une version sommaire de ce texte a été publiée sous le titre «L'identité sectaire des Esséniens » (Duhaime 2003b). 
Le dualisme qumranien s'inscrit parfaitement dans cette logique (voir Duhaime 2000a). Il peut être compris comme l'un des outils conceptuels de «resocialisation » employés par un groupe - ou plusieurs groupes apparentés - pour faire passer ses membres d'un univers symbolique à un autre et promouvoir l'adoption d'un comportement adapté à cette nouvelle vision du monde. Se déployant de manières variées en diverses circonstances, il contribue à la construction et au maintien de cette identité sectaire.

\section{Le passage dans l'alliance}

Les manuscrits de Qumrân préservent quelques descriptions de règles d'admission qui correspondent soit à des périodes différentes dans la vie d'un même groupe, soit aux pratiques de groupes apparentés (Hempel 1999). Dans sa forme la plus simple, l' «entrée dans l'alliance de Dieu » (1QS v 8 ; voir vi 14-15; CD xv 5) est signifiée par un engagement solennel, exprimé par serment, à « retourner à la Loi de Moïse de tout son cœur et de tout son être ». En 1QS v 7-13, ce «retour» implique la soumission aux prêtres fils de Sadoq, détenteurs d'une révélation particulière concernant la Loi qui, pour cette raison, sont les authentiques gardiens de l'alliance. Par son serment d'allégeance, le volontaire s'oblige également à une coupure radicale avec ceux du dehors:

Quiconque entre au Conseil de la Communauté, (il) entrera dans l'alliance de Dieu à la vue de tous les volontaires. Il s'engagera, par un serment d'obligation sur sa personne, à se convertir à la Loi de Moïse, selon tout ce qu'il a commandé, du fond de son cœur et de son être, d'après tout ce qui en a été révélé aux Fils de Sadoq, les prêtres qui gardent l'alliance et cherchent ce qui lui plaît, et d'après l'ensemble des hommes de leur alliance qui sont ensemble volontaires pour (accomplir) sa vérité et marcher selon ce qui lui plaît. Il s'engagera par alliance, sur sa personne, à se séparer de tous les hommes de perversion qui marchent dans les voies de la méchanceté. Ils ne peuvent compter dans son alliance, puisqu'ils ne l'ont pas recherché et ne l'ont pas consulté, à travers ses décrets, pour connaitre les choses cachées en quoi ils se sont égarés, se rendant coupables, et les choses révélées en quoi ils ont agi avec arrogance, excitant (ainsi) la colère du jugement et l'exercice de la vengeance par les imprécations de l'alliance. Contre eux (Dieu) exécutera de grands jugements (aboutissant) à une destruction éternelle, sans reste. (1QS v 7-13)

Selon une version plus élaborée de la procédure d'admission (1QS vi 13b-23), l'intégration du nouveau membre se fait progressivement. Les capacités et la conduite du candidat sont évaluées, d'abord par le chef des 
« Nombreux », puis par l'ensemble du groupe, pour s'assurer qu'il est apte à recevoir la formation. S'il est accepté, il s'engage alors dans une période probatoire de deux années sanctionnées par une évaluation de mi-parcours et une décision finale. Son cheminement ne s'arrête pourtant pas là, puisqu'on procède à un classement annuel des membres, au mérite:

Ainsi entrera-t-il dans l'alliance pour agir conformément à tous ces décrets pour la Communauté (qui est) une sainte Congrégation. Ils examineront leurs esprits en commun, (distinguant) chacun de son prochain, selon sa compréhension et ses œuvres dans la Loi, sous l'autorité des Fils d'Aaron qui sont volontaires dans la Communauté pour établir son alliance et pour observer tous ses décrets, qu'il a commandé d'accomplir, et sous l'autorité de la multitude d'Israël qui est volontaire pour revenir à son alliance grâce à la Communauté. Ils les inscriront en ordre, chacun devant son prochain, selon sa compréhension et ses œuvres. Chacun obéira à son prochain, l'inférieur au supérieur. Ils passeront en revue leur esprit et leurs œuvres année après année, pour élever chacun selon sa compréhension et la perfection de sa voie, ou le rétrograder selon ses défaillances. (1QS v 20-24; voir ii 19-23 ; ix 14-16)

Le formulaire de la cérémonie annuelle d'alliance, qu'on lit au début de la Règle de la communauté, est encore plus explicite sur l'idéologie de la secte et la délimitation de ses frontières. Il s'ouvre par un énoncé des objectifs du groupe dans lequel s'articule la construction bipolaire de la réalité:

... chercher Dieu de tout son cœur et de tout son être, faire tout ce qui est bon et droit en sa présence $[\ldots]$; aimer tout ce qu'il a choisi et [...] détester tout ce qu'il a rejeté, s'éloigner de tout mal et s'attacher à toutes les bonnes œuvres pour agir en justice, vérité et rectitude [...]; aimer tous les fils de lumière, chacun selon son lot dans le conseil de Dieu, et détester tous les fils de ténèbres, chacun selon sa culpabilité [...]. (1QS i 1-11a)

Puis, dans deux récits parallèles (1QS i 21 - ii 1a), prêtres et lévites proposent une interprétation de l'histoire conçue comme une suite d'actions justes, puissantes et miséricordieuses de Dieu à l'endroit d'Israël, auxquelles répondent «les iniquités des fils d'Israël et toutes leurs fautes coupables et leurs péchés ", signe qu'ils sont sous le joug de Bélial, le maître surnaturel des forces des ténèbres. "Ceux qui passent dans l'alliance " sont invités à s'identifier à cet Israël pécheur, sans doute pour mieux souligner leur volonté de changement:

[Et tou]s ceux qui passeront dans l'alliance confesseront après eux en disant: «Nous avons péché, nous avons commis des transgressions et des fautes, nous avons été méchants, nous [et] nos [pè]res avant nous, par notre comportement 
[...] (Il est) vrai et just[e, (le) Dieu d'Israël et] son jugement contre nous et contre [nos] pères, mais il exerce sur nous la miséricorde de sa grâce, depuis toujours et pour toujours. » (1QS i 24 - ii 1a)

Une bénédiction des «hommes du lot de Dieu» (1QS ii 1b-10) et une malédiction des «hommes du lot de Bélial », sanctionnées par un double «Amen» des participants, accentuent encore davantage la démarcation entre les deux univers:

Qu'il te bénisse de tout bien et te garde de tout mal. Qu'il illumine ton cœur par l'intelligence de vie et te favorise de la connaissance éternelle. Qu'il lève vers toi le visage de sa grâce pour (que tu aies) la paix éternelle. (1QS ii 2-4) Malheur à toi en toutes tes œuvres méchantes, coupables. Que Dieu te livre (à) la terreur par les agents de vengeance. Qu'il te punisse de destruction par tous ceux qui dispensent les rétributions. Malheur à toi, sans miséricorde, selon les ténèbres de tes œuvres. Damné sois-tu dans l'obscurité du feu éternel. Que Dieu ne te favorise pas quand tu crieras vers lui. Qu'il ne te pardonne pas en effaçant tes péchés. Qu'il lève le visage de sa colère pour se venger de toi et qu'il n'y ait aucune (parole) de paix pour toi dans la bouche de tous les intercesseurs. (1QS ii 5-9)

Une dernière malédiction (1QS ii 11-18), prononcée contre celui qui simule la conversion tout en gardant devant lui l' "obstacle qui le fait trébucher », pousse l'adepte à examiner l'authenticité de sa démarche et le prépare à affronter éventuellement de faux frères dont il devra se dissocier sans pitié :

Malheur, à cause des idoles qu'il sert en son cœur, à celui qui entre dans cette alliance en mettant devant lui l'obstacle de son péché pour s'y égarer. Voici qu'en entendant les paroles de cette alliance, il se bénira en lui-même en disant: "Que je sois en paix même si je marche dans l'obstination de mon cœur ». [...] Que Dieu le mette à part, pour le mal, et qu'il soit retranché du milieu de tous les fils de lumière [...]. (1QS ii 11-16)

Répétée chaque année (1QS ii 19-25), cette cérémonie devient un moyen « routinier» pour consolider l'engagement de tous et signifier concrètement, par le rang qui est alors assigné à chacun, sa progression vers l'idéal proposé:

Ainsi feront-ils année après année, tout le temps de la domination de Bélial. Les prêtres passeront en premier dans l'ordre, selon leurs esprits, l'un après l'autre. Et les lévites passeront après eux, et tout le peuple passera en troisième dans l'ordre, l'un après l'autre, par milliers, centaines, cinquantaines et dizaines, pour que chaque Israélite connaisse le rang qu'il tient dans la 
Communauté de Dieu pour un Conseil éternel. Et nul ne descendra du rang qu'il tient ou ne s'élèvera du lieu (où se trouve) son lot. (1QS ii 19-23)

\section{Le pur et l'impur}

La rhétorique du pur et de l'impur, autre façon de structurer la réalité de façon bipolaire (Douglas 1981), est omniprésente dans les textes de Qumrân. Elle apparaît avec insistance à la fin de la section sur la cérémonie d'alliance (1QS ii 25b - iii 12). Celui qui persiste dans l' «endurcissement de son cœur » et n'a pas « la force de convertir sa vie » ne peut s'agréger à la communauté. Il demeure impur, tel un lépreux, tant qu'il méprise les décrets divins et refuse de se laisser instruire par la communauté:

À la source des parfaits, il n'est pas compté. Il ne sera pas nettoyé par l'expiation, ni purifié par les eaux lustrales, ni sanctifié dans les mers ou les fleuves, ni purifié par toutes les eaux d'ablution. Impur, il reste impur, tant qu'il rejette les jugements de Dieu, en sorte qu'il ne peut être corrigé dans la Communauté de son conseil. (1QS iii 3-6)

C'est en effet dans la communauté seulement qu'on peut trouver «l'esprit du vrai conseil de Dieu» et «l'esprit saint» qui permettent d'expier les fautes et de purifier toutes les iniquités, dans un «esprit droit et humble». Sans une entière adhésion de la personne aux lois divines, précise-t-on, sa chair ne peut être purifiée:

Car c'est par l'esprit du vrai conseil de Dieu que les voies de l'homme — tous ses péchés - sont effacés, pour qu'il perçoive la lumière de vie. C'est par l'esprit saint de la communauté en Sa vérité qu'il peut être purifié de tous ses péchés. C'est par un esprit droit et humble que sa faute peut être effacée. C'est par l'humilité de sa personne à l'égard de tous les décrets divins que sa chair peut être purifiée, par l'aspersion d'eaux lustrales, et en se sanctifiant par des eaux de purification. (1QS iii 6-9)

Colleen M. Conway (2000) observe que l'agencement de ce passage permet au candidat, vraisemblablement au terme de son initiation, de faire un aller-retour imaginaire sur la voie de la dissension. La contemplation virtuelle de l'infamie à laquelle il s'exposerait dans le monde extérieur ne peut que raffermir sa décision de se soumettre à la stricte discipline du groupe détenteur de la connaissance nécessaire à sa purification physique et morale : «En évoquant les notions familières de pureté et de pollution et en les insérant dans un contexte nouveau, conclut Conway, ce texte encourage 
subtilement le lecteur à substituer, volontairement ou non, sa catégorisation antérieure du monde à celle de Qumrân » $(2000,120)$.

\section{Les deux esprits}

La longue catéchèse sur les esprits (1QS iii 13 - iv 26) est destinée à l'«Instructeur» (maśkyl) pour l'enseignement de «tous les fils de lumière ${ }^{3}$. Elle s'ouvre (1QS iii 13 - iv 1) par l'affirmation que le "Dieu des connaissances ", de qui tout vient, a créé l'homme pour dominer le monde. Pour le guider, il l'a mis en présence des esprits de vérité et de perversion, associés respectivement à la lumière et aux ténèbres et à leurs chefs surnaturels, le prince de lumières et l'ange de ténèbres. Les égarements et déboires des fils de justice sont interprétés comme des assauts des forces du mal, mystérieusement intégrés au plan de Dieu. Bien qu'il soit ultimement responsable de leur existence, Dieu a cependant des sentiments contraires d'amour et de haine à l'égard des esprits de lumière et de ténèbres:

Du Dieu des connaissances (vient) tout ce qui est et sera [...]. Il a créé l'humanité pour la domination du monde. Et il lui a mis deux esprits pour se conduire selon eux jusqu'au moment de sa visite. Voici les esprits: la vérité et la perversion. [...] Dans une source de lumière (sont) les origines de la vérité et d'une fontaine de ténèbres (sont) les origines de la perversion. Et dans la main d'un prince de lumières (est) la domination de tous les fils de justice et (c'est) selon les voies de lumière (qu')ils se conduisent. Et dans la main d'un ange de ténèbres (est) toute domination sur les fils de perversion et (c'est) selon les voies de ténèbres (qu')ils se conduisent. Et à cause de l'ange de ténèbres est l'égarement de tous les fils de justice [...] et tous les esprits de son lot (cherchent) à faire trébucher les fils de lumière. Mais le Dieu d'Israël et son ange de vérité (viennent en) aide à tous les fils de lumière. Et lui a créé les esprits, lumière et ténèbres [...]. L'un, Dieu l'aime pour tous les [mo]ments éternels $[. .$.$] ; l'autre, il (le) déteste beaucoup et toutes ses voies il les hait à$ perpétuité. (1QS iii 15 - iv 1)

Sur cet arrière-plan cosmologique, la section centrale de l'instruction (1QS iv 2-14) dévoile les "conseils» des deux esprits et les sanctions respectives des deux voies qu'ils proposent. Sa structure parfaitement balancée met en opposition deux types de personnalité et de comportement (voir Duhaime 2000b). Le fils de vérité est essentiellement centré sur Dieu, ses préceptes et ses actions; il se caractérise principalement par des attitudes

3. Voir à ce sujet Duhaime 2003a. J'utilise la traduction parue dans cet essai. 
telles que l'humilité, la perspicacité, la pureté, la discrétion et le zèle pour l'action droite:

Voici leurs voies dans le monde: pour illuminer le cœur de l'homme, pour aplanir devant lui toutes les voies de vraie justice, et pour faire craindre (à) son cœur les jugements de Dieu : un esprit d'humilité, de lenteur à la colère, de grande miséricorde, de bonté constante [...]. De la foi en toutes les actions de Dieu et l'appui sur sa grande affection: [...] un zèle pour les justes jugements, un plan saint dans un penchant ferme, une grande affection pour tous les fils de vérité [...], une discrétion pour la vérité des mystères de connaissance. (1QS iv 2-6a)

$\mathrm{Au}$ contraire, quiconque suit l'esprit de perversité est totalement centré sur lui-même et néglige «le service de la justice »; chez lui, tout n'est que méchanceté, mensonge, débauche, souillure et aveuglement:

Mais pour l'esprit de perversité, ambition personnelle, relâchement des mains dans le service de la justice: méchanceté et mensonge, orgueil et hauteur de cœur, fausseté, tricherie cruelle grande hypocrisie, prompte colère, grande folie et zèle insolent. Des actions abominables dans un esprit de débauche et des voies souillées dans un service d'impureté: une langue blasphématoire, aveuglement des yeux et dureté d'oreille, raideur de nuque et dureté de cœur, pour se conduire selon toutes les voies de ténèbres et de ruse maligne. (1QS iv 9-11a)

Le premier est promis à une existence heureuse en ce monde et glorieuse dans l'au-delà :

Voilà les conseils de l'esprit aux fils de vérité du monde. Et la visite de tous ceux qui se conduisent selon lui (sera) pour une guérison, une grande paix dans une longueur de jours et une postérité florissante avec toutes les bénédictions durables, et une joie éternelle dans la vie perpétuelle et une couronne de gloire avec un vêtement d'honneur dans la lumière éternelle. (1QS iv $6 \mathrm{~b}-8)$

Celui qui se laisse guider par l'esprit de perversité, au contraire, ne peut anticiper que douleurs et malheurs jusqu'à son extermination définitive:

Et la visite de tous ceux qui se conduisent selon lui (sera) pour un grand (nombre de) coups par la main de tous les anges de destruction, pour la perdition éternelle par l'ardeur de la colère vengeresse de Dieu, pour une terreur perpétuelle et une honte durable avec l'opprobre de l'extermination par le feu des ténèbres. Et tous leurs temps pour leurs générations (s'écoulera) dans l'affliction douloureuse et le malheur amer dans les calamités de ténèbres 
jusqu'à leur extermination pour (qu'il ne subsiste) aucun reste et aucun rescapé pour eux. (1QS iv 11b-14)

La dernière section (iv 15-26) précise que la vérité et la perversité sont incompatibles et se livrent une lutte féroce, à laquelle les humains prennent part, "jusqu'au moment ultime ». Le théâtre de ce combat est non seulement l'univers, mais également le cœur de chaque homme, selon son partage entre vérité et perversion: «Dieu les a placées une partie contre l'autre jusqu'au temps ultime. Il a établi une haine éternelle entre leurs catégories: abomination (pour la) vérité, les actions de perversité et abomination (pour la) perversité tous les chemins de vérité. » (1QS iv 16-17) Toutefois, au terme fixé par Dieu, la perversion sera éliminée totalement et ceux qu'il a choisis pour «l'alliance éternelle» se verront totalement purifiés, connaîtront la «sagesse des fils du ciel » et seront revêtus de «la gloire d'Adam »:

Mais Dieu, dans les secrets de sa science et dans la sagesse de sa gloire, a établi un temps pour l'existence de la perversité. Au moment de sa visite, il l'éliminera à jamais. [...] Alors Dieu nettoiera dans sa vérité toutes les œuvres de l'humain [...] pour le purifier dans l'esprit de sainteté de toutes ses activités d'impiété. Il fera jaillir sur lui l'esprit de vérité [...] pour faire comprendre aux droits la connaissance du Très-Haut, et pour que la sagesse des fils du ciel soit apprise aux parfaits de conduite, car Dieu les a choisis pour l'alliance éternelle, et pour eux (sera) toute la gloire d'Adam. [...] Jusqu'à maintenant les esprits de vérité et de perversion combattent dans le cœur de l'humain: ils se conduisent avec sagesse et folie [...]. Car (c'est) une partie contre l'autre (que) Dieu les a placées jusqu'au temps de la décision et de l'agir nouveau. (1QS iv 18-25)

Dans sa forme actuelle, cette instruction propose avant tout une éthique communautaire, mais elle la situe dans une vision globale du monde qui en trace les origines et l'aboutissement, lui conférant ainsi sens et plausibilité. On retrouve encore ici les rhétoriques de la division et de la distinction qui contribuent à forger l'identité sectaire en immergeant totalement l'individu dans un univers dualiste «non seulement au niveau d'une proposition intellectuelle, mais à celui de la perception spontanée» (Newsom 1990, 41).

\section{Les Hymnes comme récits identitaires}

Plongé dans la représentation de l'univers, de l'histoire et de l'être humain que le groupe lui propose, l'adepte peut réinterpréter son passé à la lumière 
de son statut actuel. La plupart des mouvements sectaires connaissent ce phénomène, qui prend souvent la forme d'un récit de vie ou de conversion. André Billette (1975) a montré que de tels récits sont élaborés à partir d'une sélection d'événements réels dont le sens, dévoilé rétrospectivement à l'aide des cadres d'analyse du groupe, s'exprime selon des formes stéréotypées. L'adepte identifie habituellement un «point tournant » dans son expérience de conversion, à partir duquel se découpent un «avant » et un «après ». Le témoignage permet alors d'affirmer publiquement et de consolider son engagement, tout en fournissant aux nouveaux membres un modèle dont ils s'inspirent à leur tour.

Sans être identiques à ces récits, plusieurs Hymnes de Qumrân semblent jouer un rôle analogue dans la construction de l'identité sectaire. Carol A. Newsom a observé comment trois thèmes principaux s'y entrecroisent (2001; 2004). Les Hymnes se présentent avant tout comme des prières où le fidèle, peut-être un chef spirituel du groupe et un modèle pour ses membres, rend grâce à Dieu d'avoir été placé dans une communauté de salut à laquelle ont été révélés des mystères de connaissance apparentés au contenu de l'Instruction sur les deux esprits: "Dans ta colère sont tous les châtiments, mais dans ta bonté est l'abondance des pardons; et tes tendresses sont pour tous les fils de ton bon plaisir car tu leur as fait connaître le secret de ta vérité et tu les as instruits de tes mystères merveilleux.» $\left(1 \mathrm{QH}^{\mathrm{a}}\right.$ vi [xi] 8-10)

L'orant affirme également sa conscience de n'être qu'une créature d'argile, portée au péché; il ne doit son salut qu'à la grâce de Dieu et il a sans cesse besoin d'être corrigé :

Ces choses, je les ai connues grâce à ton intelligence, car tu as révélé à mes oreilles tes mystères merveilleux, moi qui ne suis qu'un modelage d'argile, pétri avec de l'eau, une base de honte, une source d'impureté, un four à iniquité et une bâtisse de péché, un esprit d'erreur et de dépravation, sans intelligence et effrayé par les justes jugements. $\left(1 \mathrm{QH}^{\mathrm{a}}\right.$ ix [i] 21-23)

Enfin, plusieurs hymnes situent le fidèle au cœur d'un affrontement avec les forces des ténèbres et de la perversion qui déferlent sur lui: "Je te rends grâces, Seigneur, car tu m'as placé dans le sachet de vie et tu m'as protégé contre tous les pièges de la fosse, car des agresseurs voulaient s'en prendre à moi parce que je m'appuyais sur ton alliance...» $\left(1 \mathrm{QH}^{\mathrm{a}} \mathrm{x} 20-22\right)$

Mais son attachement persévérant à l'alliance et sa confiance absolue en Dieu finissent toujours par avoir raison de ces épreuves. 
En développant ces trois thèmes sous diverses formes, les Hymnes contribuent à renforcer chez le membre la prétention de la communauté à posséder le savoir qui conduit au salut, tout en le disposant à l'ascèse et à la discipline, tant par nécessité de combattre la propension humaine au mal que pour résister aux assauts des «ennemis ».

\section{La guerre eschatologique}

Selon le sociologue Georg Simmel, les conflits qu'un groupe entretient avec l'extérieur sont souvent un facteur de cohésion interne: ils contribuent à clarifier et à raffermir les relations entre ses membres, à rehausser leur degré d'engagement et à éliminer toute dissension. Simmel estime qu'il est même parfois "politiquement habile ", à l'intérieur d'un groupe, "de veiller à ce que l'on ait des ennemis » (1992, 115-125).

La Règle de la guerre et d'autres documents qumraniens apparentés contribuent largement à la construction et au maintien d'une idéologie de conflit susceptible de favoriser la socialisation des membres du ou des groupes qui les ont composés ou en ont fait usage. Telle que préservée dans la grotte 1, la Règle de la guerre (1QM) anticipe une lutte à finir entre les fils de lumière et les fils de ténèbres (col. i), avec ses préparatifs tactiques (col. ii - ix), ses prières (col. $\mathrm{x}$ - xiv) et ses diverses phases, culminant dans le triomphe des Fils de lumière, soutenus par les troupes célestes (col. xv - xx) (voir Duhaime $1995 ; 2004 ; 2006)$.

Cette guerre sera une période de "grande détresse ", mais qui conduira à une « rédemption éternelle»:

Ce sera un temps de détresse su[r tou]t le peuple de la rédemption de Dieu; dans toutes leurs détresses, il n'y en a pas eu comme celle-là, à cause de sa hâte vers sa consommation pour la rédemption éternelle. (1QM i 12)

Il y aura probablement des victimes parmi les fils de lumière, surtout lorsque Bélial, le chef céleste des forces du mal, viendra au secours des fils des ténèbres; mais cela fait partie des mystères de Dieu qui "éprouve le cœur de son peuple au creuset », selon l'image employée dans le discours d'exhortation du prêtre en chef:

Lorsque [Bé]li[al] se ceindra pour aider les fils de ténèbres et que les tués des (hommes) d'infanterie commenceront à tomber, par les mystères de Dieu et pour éprouver en eux tous ceux qui ont été désignés pour le combat, les prêtres son[neront dans les trom]pettes de l'appel pour faire sortir une autre ligne (comme) relève pour le combat [...]. Le prêtre en chef s'approchera [...] et il 
affermira leur cœur dans [les prouesses de Dieu et] leurs [main]s dans la guerre. Il prendra la parole pour dire : « [... le Dieu d'Israël se lève et le] cœur de son peuple, il l'éprouve au creuset [...] et il placera leur salut dans le feu [...]. Il aiguisera les éprouvés du creuset en armes de guerres, et elles ne s'émousseront pas jusqu'à [l'extermination éternelle de toutes les nations] impies. (xvi 11 - xvii 2)

C'est pourquoi, dans le combat d'une ampleur sans précédent qui doit conduire à l'anéantissement définitif des forces du mal, les "fils de l'alliance » sont exhortés à tenir bon jusqu’à la victoire définitive:

Et vous, montrez-vous forts et ne craignez pas [...]. Car au Dieu d'Israël (est) tout ce qui a été et [ser]a dans tous les devenirs éternels. Ce jour (est) son moment pour humilier et abaisser le prince de la domination de l'impiété. [...] Et vous, fils de son alliance, montrez-vous fort dans le creuset de Dieu, jusqu'à ce qu'il agite sa main et remplisse ses creusets en ses secrets pour que vous teniez debout. (1QM xvii 9)

Les opinions divergent sur la provenance et l'usage d'un pareil document. Il est toutefois vraisemblable que certaines de ses prescriptions aient servi de modèles à un groupe sectaire, comme ce fut sans doute le cas pour la Règle de la congrégation (Schiffman 1989). En plus de distinguer nettement les deux camps opposés de la lumière et des ténèbres, ce texte entretient l'idée de l'imminence de leur affrontement dans une bataille aux proportions cosmiques, dont l'issue est déjà décidée. Avec son caractère utopique et eschatologique, cette vision des choses pouvait conforter les membres d'un groupe sectaire dans leur décision de rompre complètement avec un environnement considéré comme perverti, pour vivre dans une stricte obéissance à la Loi de Moïse; elle offrait également une explication à d'éventuelles défections de la part de ceux qui ne réussiraient pas à supporter les assauts répétés des forces du mal, véritable creuset qui "épure » le fidèle. Il n'est pas exclu que cette Règle ait incité ses lecteurs à prendre part au soulèvement contre les Romains et leurs alliés, identifiés au mal par excellence; mais cela est indémontrable.

\section{Conclusion}

Ce rapide survol laisse entrevoir comment le développement de croyances dualistes et leur déploiement dans divers registres de discours et de pratiques ont pu contribuer à reconfigurer l'univers symbolique et l'identité juive dans une perspective sectaire vers la fin de la période du deuxième Temple. Les 
groupes dont témoignent les textes de Qumrân avaient également d'autres moyens à leur disposition, tels que l'étude et l'interprétation communautaire de l'Écriture ainsi que l'élaboration de règles disciplinaires et de doctrines particulières (messianisme, communion avec les anges, etc.). La perspective des sciences sociales permet souvent de mieux identifier ces stratégies de construction identitaire et de les comprendre en les mettant en relation avec des modèles et des théories validées sur un abondant matériel comparatif. Elle apporte ainsi une contribution originale et non-négligeable aux autres approches des textes de Qumrân, tout comme elle le fait depuis un certain temps dans le domaine biblique. Surtout, elle permet de démontrer que la transformation des croyances ne résulte pas seulement de pures spéculations intellectuelles ou mystiques, mais qu'elle est étroitement liée à la dynamique socioreligieuse des groupes qui les produisent.

\section{Références}

Berger, P.L. et T. LuckMANN (1996) [1986, anglais 1966], La construction sociale de la réalité / trad. par P. Taminiaux, Paris, Colin (Références).

Billette, A. (1975), Récits et réalités d'une conversion, Montréal, Presses de l'Université de Montréal.

CARmignaC, J. et al. (1961-1963), Les textes de Qumrân. Traduits et annotés, I-II, Paris, Letouzey et Ané.

Charlesworth, J.H., dir. (1996), The Dead Sea Scrolls: Rule of the Community. Photographic Multi-Language Edition, Philadelphia, American Interfaith Institute.

Conway, C.M. (2000), "Toward a Well-Formed Subject: The Function of Purity Language in the Serek Ha-Yahad ", Journal for the Study of the Pseudepigrapha, 21, p. 103-120.

Douglas, M. (1981) [1971, anglais 1966], De la souillure. Essai sur les notions de pollution et de tabou / trad. par A. Guérin, Paris, Maspéro.

Duhaime, J. (1995), «War Scroll», dans J.H. Charlesworth, dir., The Dead Sea Scrolls: Hebrew, Aramaic, and Greek Texts with English Translations, vol. 2: Damascus Document, War Scroll and Related Documents, Tübingen / Louisville, J.C.B. Mohr (Paul Siebeck) / Westminster John Knox Press (The Princeton Theological Seminary Dead Sea Scrolls Project), p. 80-141. 
— (2000a), "Dualism», dans L.H. Schiffman et J.C. VAnderKam, dir., Encyclopedia of the Dead Sea Scrolls, New York, Oxford University Press, p. 215220.

- (2000b), «Les voies des deux esprits (1QS iv 2-14). Une analyse structurelle ", Revue de Qumrân, 19, p. 349-369.

- (2003a), "Cohérence structurelle et tensions internes dans l'Instruction sur les deux esprits (1QS III 13 - IV 26) ", dans F. GarCía-MarTínEZ, dir., Wisdom and Apocalypticism in the Dead Sea Scrolls and the Biblical Tradition, Louvain, Peeters / Leuven University Press (Bibliotheca Ephemeridum Theologicarum Lovaniensium 168), p. 103-131.

— (2003b), «L'identité sectaire des Esséniens », Le Monde de la Bible, 151, p. 43-47.

- (2004), The War Texts: 1QM and Related Manuscripts, London / New York, T\&T Clark (Companion to the Qumran Scrolls 6).

— (2006, à paraître), "La Règle de la Guerre (1QM) et la construction de l'identité sectaire", dans F. García-MarTínez, dir., Defining Identities: Who Is the Other? We, You and the Others in the Dead Sea Scrolls, Leiden, Brill.

Dupont-Sommer, A. et M. Philonenko, dir. (1987), La Bible. Écrits intertestamentaires, Paris, Gallimard (Bibliothèque de la Pléiade).

Hempel, C. (1999), "Community Structures in the Dead Sea Scrolls: Admission, Organization, Disciplinary Procedures ", dans P.W. FuINT et J.C. VanderKam, dir., The Dead Sea Scrolls After Fifty Years : A Comprehensive Assessment, vol. 2, Leiden, Brill, p. 67-92.

Jokiranta, J.M. (2001), "Sectarianism” of the Qumran "Sect" ", Revue de Qumrân, 20, p. 223-239.

Newsom, C.A. (1990), "Apocalyptic and the Discourse of the Qumran Community ", Journal of Near Eastern Studies, 49, p. 135-144.

- (2001), «Apocalyptic Subjects: Social Construction of the Self in the Qumran Hodayot ", Journal for the Study of the Pseudepigrapha, 12/1, p. 3-35.

- (2004), The Self as Symbolic Space: Constructing Identity and Community at Qumran, Leiden, Brill (Studies on the Texts of the Desert of Judah 52).

Schiffman, L.H. (1989), The Eschatological Community of the Dead Sea Scrolls, Atlanta, Scholars Press.

Simmel, G. (1992) [allemand 1908], Le conflit / trad. par S. Muller, Saulxures, Circé. Une traduction légèrement différente se trouve dans G. SIMMEL, Sociologie. Études sur les formes de la socialisation, Paris, Presses Universitaires de France (Sociologie), 1999, p. 265-346. 
Wise, M., M. Abegg Jr et E. Cook (2001), Les manuscrits de la mer Morte, Paris, Plon; réédition : Paris, Perrin, 2003.

\section{Résumé}

Plusieurs textes de Qumrân sont fortement imprégnés d'une idéologie dualiste. Empruntant aux théories et modèles des sciences sociales, cette étude examine la contribution d'une telle idéologie à la structuration de l'identité sectaire dans le ou les groupes dont ils sont le reflet. Après des précisions sur le concept d'univers symbolique et la place du dualisme qumranien dans ce cadre général, l'auteur analyse sommairement les règles d'admission, l'opposition entre le pur et l'impur et l'affrontement des deux esprits selon la Règle de la communauté, les récits identitaires des Hymnes et la représentation du combat ultime de la lumière contre les ténèbres dans la Règle de la Guerre.

\section{Abstract}

Several scrolls from Qumran bear a strong dualistic ideology. Based on social sciences theories and models, this study explores the contribution of such an ideology to the construction of the sectarian identity in the group or groups which they mirror. Having defined the concept of symbolic universe and located the dualism from Qumran within this larger context, the author proceeds to a summary examination of the admission rules, the opposition between purity and impurity, and the conflict between the two spirits according to the Rule of the Community, the identity narrative found in the Hymns, and the representation of the ultimate battle between light and darkness according to the War Scroll. 